\title{
The Glauber dynamics on colourings of a graph with high girth and maximum degree
}

\author{
Michael Molloy* \\ Department of Computer Science \\ University of Toronto \\ Toronto, Canada
}

July 24, 2003

\begin{abstract}
We prove that the Glauber dynamics on the $C$-colourings of a graph $G$ on $n$ vertices with girth $g$ and maximum degree $\Delta$ mixes rapidly if (i) $C=q \Delta$ and $q>q^{*}$ where $q^{*}=1.4890 \ldots$ is the root of $\left(1-\mathrm{e}^{-1 / q}\right)^{2}+q \mathrm{e}^{-1 / q}=1$; and (ii) $\Delta \geq D \log n$ and $g \geq D \log \Delta$ for some constant $D=D(q)$. This improves the bound of roughly $1.763 \Delta$ obtained by Dyer and Frieze [2] for the same class of graphs. Our bound on this class of graphs is lower than the bound of $11 \Delta / 6 \approx$ $1.833 \Delta$ obtained by Vigoda [13] for general graphs.
\end{abstract}

For a given graph $G$ and integer $C$ which is at least the chromatic number of $G$, we define the Glauber dynamics on the $C$-colourings of $G$ to be the Markov chain described as follows. We start with an arbitrary $C$-colouring, and at each step we choose a uniformly random vertex $v$, and a uniformly random colour $c$ from $L(v)$, the list of colours which do not appear on any

*This work is supported by an NSERC Research Grant and a Sloan Research Fellowship. 
neighbours of $v$. Then we change the colour of $v$ to $c$. (If $L(v)$ is empty then we do not choose a colour; note that $L(v)$ is never empty if $C>\Delta$.)

Unless specified otherwise, we consider all colourings to be proper, i.e. no two adjacent vertices can have the same colour. In section 3 , it will be important to note that we can apply this step even to a non-proper colouring of $G$. But note that if a colouring is proper, then applying a step of the chain cannot produce an improper colouring.

The main question in this area is: For what values of $C$ does this Markov chain mix in polytime? Usually this is studied in terms of $\Delta$, the maximum degree of $G$. It is well known that for some graphs, the chain does not mix for $C \leq \Delta+1$. (In fact, there are some graphs and $(\Delta+1)$-colourings for which no colour changes are possible, and so the chain is not even ergodic.) Jerrum [7] showed that for all graphs, the chain mixes in polytime for $C \geq 2 \Delta$ and in optimal time, i.e. $O(n \log n)$ time, for $C \geq 2 \Delta+1$. Salas and Sokal[11] independently obtained the latter result. Vigoda [13] showed that for all graphs, a different chain mixes in optimal time for $C \geq \frac{11}{6} \Delta$ and this implies that for the same values of $C$, the Glauber dynamics mixes in polytime. Dyer, Greenhill and Molloy [5] showed that the Glauber dynamics mixes in optimal time for $C \geq(2-\epsilon) \Delta$ where $\epsilon$ is a small positive constant (see also [9]). Some work has been done on special classes of graphs. Dyer et al[3] showed that the Glauber dynamics mixes in optimal time on triangle-free graphs when $C \geq(2-x) \Delta$ for a different small positive constant $x$. More recently, Dyer and Frieze[2] showed that if the maximum degree, $\Delta$, of $G$ is at least $D \log n$ and the girth is at least $D \log \Delta$ for some sufficiently large constant $D$, then the chain mixes in optimal time for $C \geq q \Delta$ for any constant $q>\beta$ where $\beta=1.763 \ldots<11 / 6$ is the root of $\beta \mathrm{e}^{-1 / \beta}=1$. Here we improve on this latter result:

We define $q^{*}=1.4890 \ldots$ to be the root of $\left(1-\mathrm{e}^{-1 / q}\right)^{2}+q \mathrm{e}^{-1 / q}=1$.

Theorem 1 For any $q>q^{*}$ and integer $\omega$, there exists $D$ for which: Suppose $G$ has $n$ vertices, maximum degree $\Delta \geq D \log n$ and no vertex lies in more than $\omega$ cycles of length less than D $\log \Delta$. Then the Glauber dynamics mixes in time $O(n \log n)$ for $C=q \Delta$.

Of course, this covers all graphs with girth at least $D \log \Delta$. It also covers natural models of random graphs such as $G_{n, p=c / n}$ for $c \approx D \log n$ and 
random $\Delta$-regular graphs for $\Delta=D \log n$. Dyer and Frieze[2] showed that their theorem also extends to such graphs.

Throughout the paper, we assume that $n$ is large enough for various asymptotic bounds to hold. We can also assume that $q$ is sufficiently close to $q^{*}$. We use $N(v)$ to denote the neighbourhood of $v$, i.e. the set of vertices which are adjacent to $v$. We define $d(v)=|N(v)|$ to be the degree of $v$. A short cycle is a cycle of length less than $D \log \Delta$.

\section{Some intuition}

The proofs of all results mentioned above, except for that of Vigoda [13], all come from the following idea ${ }^{1}$. Consider two colourings $X, W$ which differ only at one vertex $v$. We will carry out one step of the process on each colouring, where we couple these two random steps maximally. Specifically, we first choose a uniform vertex $u$ for both colourings. If $L_{X}(u)$ and $L_{W}(u)$ are the sets of allowable colours for $u$ in $X, W$ respectively, then we take two mappings $f_{X}:[0,1] \rightarrow L_{X}(u), f_{W}:[0,1] \rightarrow L_{W}(u)$, such that

- for each $c \in L_{X}(u),\left|f_{X}^{-1}(c)\right|=1 /\left|L_{X}(u)\right|$ and similarly for $W$, and

- $\left\{x: f_{X}(x) \neq f_{W}(x)\right\}$ is as small as possible.

Then we take a uniform random real $x \in[0,1]$ and choose $u, f_{X}(x)$ for $X$ and $u, f_{W}(x)$ for $W$. Note that since $L_{X}(u), L_{W}(u)$ differ in at most one colour per list, we will have $\left|\left\{x: f_{X}(x) \neq f_{W}(x)\right\}\right| \leq \min \left\{\left|L_{X}(u)\right|^{-1},\left|L_{W}(u)\right|^{-1}\right\}$. Note further that $L_{X}(u)=L_{W}(u)$ unless $u$ is a neighbour of $v$.

Using the path-coupling technique of Bubley and Dyer [1], it suffices to show that the probability of $X, W$ converging after one step is greater than the probability of them differing in a second vertex after one step (we elaborate on this in Section 3). They converge iff we choose $u=v$, which occurs with probability $1 / n$. They differ on a second vertex iff we choose some $u \in N(v)$ and we choose $x \in\left\{x: f_{X}(x) \neq f_{W}(x)\right\}$. Since no list can ever be smaller than $C-\Delta$, this occurs with probability at most $\frac{\Delta}{n} \times \frac{1}{C-\Delta}$ which is less than $\frac{1}{n}$ so long as $C>2 \Delta$.

\footnotetext{
${ }^{1}$ Jerrum's original proof in [7] predated this idea, but the idea yields a simpler proof.
} 
The bound was improved slightly in [3] by showing that for trianglefree graphs, after a relatively short period, most vertices will tend to have many repeated colours in their neighbourhoods. Thus, their lists of available colours will tend to be somewhat greater than $C-\Delta$, and this leads to a gain in the above calculations. The same idea played a key role in [5].

In [2], Dyer and Frieze showed that for graphs with large girth and maximum degree, after $O(n \log n)$ steps, with high probability every vertex will have a list of size at least roughly $q \mathrm{e}^{-1 / q} \Delta$. If $X, W$ are such that all vertices have lists of this size, then this yields that the probability of $X, W$ differing on a second colour is at most $\frac{\Delta}{n} \times \frac{1}{q \mathrm{e}^{-1 / q} \Delta}$. Since $q$ is chosen so that $q \mathrm{e}^{-1 / q}>1$, this probability is less than $\frac{1}{n}$.

The key new idea used in this paper is to show that, after $O(n \log n)$ steps, many neighbours $u$ of $v$ will satisfy $L_{X}(u)=L_{W}(u)$. If we make the simplifying assumption that the graph is $\Delta$-regular, then with high probability, there will be roughly $\left(1-\mathrm{e}^{-1 / q}\right)^{2} \Delta$ such neighbours. This improves our bound on the probability of $X, W$ differing on a second colour to $\frac{\left(1-\left(1-\mathrm{e}^{-1 / q}\right)^{2}\right) \Delta}{n} \times \frac{1}{q \mathrm{e}^{-1 / q} \Delta}$, which is less than $\frac{1}{n}$ for $q>q^{*}$.

\subsection{Uniform-like colour sets}

Suppose that $G$ is $\Delta$-regular and that every vertex in $N(v)$ is assigned an independent uniform colour from $\{1, \ldots, C\}$. Then the probability that some colour $c$ does not appear on any neighbour of $v$ is $(1-1 / C)^{\Delta}=\mathrm{e}^{-1 / q}+o(1)$. Thus we would expect that each list would have size roughly $C \times \mathrm{e}^{-1 / q}=$ $q \mathrm{e}^{-1 / q} \Delta$. This explains, at least intuitively, the lower bound that Dyer and Frieze obtain.

Now suppose that every vertex of distance 2 from $v$ is also assigned an independent uniform colour from $\{1, \ldots, C\}$. Suppose further that we change the colour of $v$ in our colouring $X$ to obtain another colouring $W$. For each $u \in N(v), L_{X}(u)=L_{W}(u)$ iff the colours $X(v)$ and $W(v)$ both appear on $N(u)-v$. The probability that this occurs is $\left(1-\mathrm{e}^{-1 / q}\right)^{2}+o(1)$, and so this explains the result in this paper.

Of course, the colours appearing on the neighbours of $v$ are far from independent. But intuitively, since there are few short cycles near $v$, after 
$O(n \log n)$ steps the colours on vertices close to $v$ are "close enough" to being independent. Much of the work in this paper can be viewed as proving this statement.

\subsection{A recursive analysis}

Our situation is somewhat more complicated than that in [2]. To illustrate this, suppose that $N(v)=w_{1}, \ldots, w_{\Delta}$ and consider any assignment of colours to the vertices in $N\left(w_{1}\right), \ldots, N\left(w_{\Delta}\right)$. Now, assign to each $w_{i}$ a uniform colour from amongst those not appearing on $N\left(w_{i}\right)$, independently of the choice for any other $w_{j}$. It turns out, that for any assignment of colours to $N\left(w_{1}\right), \ldots, N\left(w_{\Delta}\right)$, the expected number of colours not appearing on $N(v)$ is at least $q \mathrm{e}^{-1 / q} \Delta-o(\Delta)$. Thus, Dyer and Frieze did not have to ensure that the random colours appearing on $N\left(w_{1}\right), \ldots, N\left(w_{\Delta}\right)$ are close to uniform. Unfortunately, when analyzing our other parameter, we do not have this advantage, and we need to prove rather tight results on the distributions of the colours appearing at distance 2 and 3 from $v$.

To do this, we require a complicated iterative analysis. We'll introduce that analysis now, in an oversimplified setting, before formalizing it in the next section. First, it will be much simpler to assume that $G$ is regular and so every vertex has degree $\Delta$. We will also pretend that the time steps are partitioned into a series of epochs, $\Psi_{0}, \Psi_{1}, \Psi_{2}, \ldots$ and that every vertex is recoloured at least exactly once during each epoch.

We will be interested in two parameters. The first is the number of colours available to each vertex. We will create a sequence $L_{0}>L_{1}>L_{2}>\ldots$ such that during epoch $\Psi_{i}$, the set $L(v)$ of colours available to vertex $v$ satisfies

$$
|L(v)| \leq L_{i}
$$

for every $v$. Furthermore, the same analysis as in [2] will yield that for $i \geq 1$, at any time during epoch $\Psi_{i}$ we have:

$$
|L(v)| \geq\left(\mathrm{e}^{-1 / q}-o(1)\right) C=q \mathrm{e}^{-1 / q} \Delta-o(\Delta) .
$$

The second parameter has to do with the probability that a particular 
colour $c$ is in $L_{v}$. For any colour $c$ and vertex $v$, we define

$$
T(v, c)=\sum_{w \in N(v), c \in L(w)} \frac{1}{|L(w)|} .
$$

Thus, if we were to choose a random colour for each neighbour of $v$ in turn, then $T(v, c)$ would be the expected number of neighbours which are assigned $c$. Furthermore, if we assume as in subsection 1.1 that these choices are independent, then the probability that no neighbours are assigned $c$, i.e. that at the end of these choices we have $c \in L(v)$ is roughly $\mathrm{e}^{-T(v, c)}$.

Of course, we need to be a bit careful here, because the sets $L(w)$ vary with time and we are actually interested in their values at different time steps. But we will overlook such details now as we are just providing an intuitive overview.

We will introduce sequences $A_{0}<A_{1}<A_{2}<\ldots$ and $B_{0}>B_{1}>B_{2}>\ldots$ such that during epoch $\Psi_{i}$, we have for every pair $v, c$ :

$$
A_{i} \leq T(v, c) \leq B_{i}
$$

We start with our recursive equation for $A_{i}$. Suppose that we are in epoch $\Psi_{i+1}$ and for each neighbour $w$ of $v$, let's pretend that the neighbours of $w$ were most recently coloured during epoch $\Psi_{i}$. At that time we had $T(w, c) \leq$ $B_{i}$ and so the probability that $c$ is now in $L(w)$ is at least roughly $\mathrm{e}^{-B_{i}}$. We also have $|L(w)| \leq L_{i}$. This inspires us to define:

$$
A_{i+1}=\Delta \times \mathrm{e}^{-B_{i}} / L_{i}
$$

Similarly, we obtain

$$
B_{i+1}=\Delta \times \mathrm{e}^{-A_{i}} /\left(\mathrm{e}^{-1 / q} C\right) .
$$

Finally, we consider $L_{i+1} \cdot \operatorname{Exp}(|L(v)|)$ is roughly $\sum_{c=1}^{C} \mathrm{e}^{-T(v, c)}$. Also, note that

$$
\sum_{c=1}^{C} T(v, c)=\sum_{w \in N(v)} \sum_{c \in L(w)} \frac{1}{|L(w)|}=\Delta .
$$

Thus, our expression for $\operatorname{Exp}(|L(v)|)$ is minimized when each $T(v, c)$ is equal to $\Delta / C$, which yields a non-recursive lower bound of $C \times \mathrm{e}^{-\Delta / C}=C \times \mathrm{e}^{-1 / q}$, as obtained in [2]. Furthermore, the expression is maximized when the values 
of $T(v, c)$ are as disparate as possible. Since each $T(v, c)$ is between $A_{i}$ and $B_{i}$, we get a recursive upper bound by assuming that every $T(v, c)$ is either $A_{i}$ or $B_{i}$. Since they sum to $\Delta$, we must have $\left(\Delta-C B_{i}\right) /\left(A_{i}-B_{i}\right)$ of them equal to $A_{i}$ and $\left(\Delta-C A_{i}\right) /\left(B_{i}-A_{i}\right)$ of them equal to $B_{i}$. That gives an upper bound of:

$$
L_{i+1}=\frac{\Delta-C B_{i}}{A_{i}-B_{i}} \times \mathrm{e}^{-A_{i}}+\frac{\Delta-C A_{i}}{B_{i}-A_{i}} \times \mathrm{e}^{-B_{i}} .
$$

After choosing appropriate initial values, it is straightforward to show that these recursive equations have a limit of $A_{i}=B_{i}=1 / q$ and $L_{i}=$ $C \times \mathrm{e}^{-1 / q}$. Therefore, by running our Markov chain for enough epochs, we can guarantee that for every $v, c$ we have $L(v)$ arbitrarily close to $\mathrm{e}^{-1 / q}$ and $T(v, c)$ arbitrarily close to $\Delta / C$. This goes a long way towards allowing us to show that the intuitive analysis outlined in subsection 1.1 holds.

To transform this intuition into a proof, we need to be much more precise. For one thing, we have to be careful about specifying the time steps at which we are measuring $L(v)$ in some of these quantities. We also can't assume that every vertex is selected exactly once per epoch; it turns out that at least once per epoch will do, and we can achieve that by taking each epoch to be of length $O(n \log n)$. Also, these recursive equations were obtained by (implicitly) assuming that in each epoch, every quantity will be equal to its expected value. In order to allow for the possibility that some quantities differ slightly from their expected values, we decrease each $A_{i}$ and increase each $B_{i}, L_{i}$ by a small amount; we also apply a concentration inequality to show that they don't differ more-than-slightly from their expected values. Finally, we can't assume that every vertex has degree exactly $\Delta$, and so we have to allow our bounds on $|L(v)|$ to be functions of the degree of $v$.

All of the adjustments outlined in the preceding paragraph are straightforward, but tedious if we assume, as in subsection 1.1, that the random colours assigned to the neighbours of a vertex are independent. Of course, this assumption is not valid and so we need to prove that they are, in some sense, close to being independent. We do this by focussing on "long-pathsof-disagreement", which will be described further in later sections. 


\section{The main lemmas}

As described in the opening part of Section 1, our goal is essentially to show that after $O(n \log n)$ steps, many neighbours $u$ of $v$ will satisfy $L_{X}(u)=$ $L_{Y}(u)$. We must complicate this condition somewhat. First, for technical reasons, we wish to make this hold independently of our choice of the colours $v$ has in $X, Y$ and so we make a statement that holds for every pair of colours $c_{1}, c_{2}$. Also, we have to adjust our goal somewhat to deal with the case where $v$ has neighbours of degree less than $\Delta$. In fact, neighbours of very small degree, less than $\rho \Delta$ for some small positive constant $\rho$ are particularly problematic and so have to be dealt with separately. Our main lemma is:

Lemma 2 For every $\epsilon, \rho>0$, there exist constants $D, \tau$ such that with probability at least $1-O\left(n^{-6}\right)$, for every vertex $v$, colours $c_{1}, c_{2}$ and time $\tau n \log n \leq t \leq n^{2}$, we have the following: Define $\theta=\theta_{c_{1}, c_{2}}(v)$ to be the set of neighbours $w$ of $v$ with $d(w) \geq \rho \Delta$ and with at least one of $c_{1}, c_{2}$ not appearing in $N(w)-v$, and define:

$$
R_{c_{1}, c_{2}}(v)=\sum_{w \in \theta} \frac{1}{|L(w)|}
$$

Then

$$
R_{c_{1}, c_{2}}(v) \leq \frac{1-\left(1-\mathrm{e}^{-1 / q}\right)^{2}}{q \mathrm{e}^{-1 / q}} \times \frac{d(v)}{\Delta}+\epsilon .
$$

Note that, under the notation of Section 1 , if $v$ has $c_{1}$ in $X$ and $c_{2}$ in $Y$, and if $d(w)>\rho \Delta$ and $L_{X}(w)=L_{Y}(w)$ then $w \in \theta_{c_{1}, c_{2}}(v)$. Thus, if the graph is $\Delta$-regular, then this lemma implies what we said our goal was in the opening part of Section 1.

In section 3, we will strengthen this lemma and then show how it implies Theorem 1. For ease of presentation, we first prove Lemma 2 and then show how to adapt the proof to yield the stronger lemma.

We begin with a recursive definition. This is along the same lines as that described in subsection 1.2, but modifed somewhat to facilitate a formal proof. For each $0 \leq d \leq \Delta$ :

$$
\text { - } \alpha_{1}=0, \beta_{1}=1 /(q-1), \lambda_{1}^{(d)}=q
$$


- $\alpha_{k+1}=\mathrm{e}^{-\beta_{k}} / \lambda_{k}^{(\Delta)}$;

- $\beta_{k+1}=\mathrm{e}^{-\alpha_{k}} /\left(q \mathrm{e}^{-1 / q}\right)$;

- $\lambda_{k+1}^{(d)}=\frac{q \beta_{k}-1}{\beta_{k}-\alpha_{k}} \mathrm{e}^{-\alpha_{k} d / \Delta}+\frac{1-q \alpha_{k}}{\beta_{k}-\alpha_{k}} \mathrm{e}^{-\beta_{k} d / \Delta}$.

Lemma $3 \lim _{k \rightarrow \infty} \alpha_{k}=\lim _{k \rightarrow \infty} \beta_{k}=1 / q, \lim _{k \rightarrow \infty} \lambda_{k}^{(d)}=q \mathrm{e}^{-d / q \Delta}$.

We postpone the proof of Lemma 3 until later. But for now, we require the following simple observation:

Lemma 4 For all $k \geq 1$ and $0 \leq d \leq \Delta$ :

(a) $\alpha_{k}<1 / q<\beta_{k}$, and

(b) $q \mathrm{e}^{-\beta_{k} d / \Delta} \leq \lambda_{k+1}^{(d)} \leq q \mathrm{e}^{-\alpha_{k} d / \Delta}$.

Proof (b) follows since $\lambda_{k+1}^{(d)}$ is a linear combination of $q \mathrm{e}^{-\beta_{k} d / \Delta}$ and $q \mathrm{e}^{-\alpha_{k} d / \Delta}$. (a) follows from a simple induction.

In subsection 1.2 we said that we have to adjust our sequences by a small amount, in order to allow for the possibility that some terms differ slightly from their expected values. We do so now:

Fix some small $\delta$ to be named later, and choose $k^{*}$ such that $\alpha_{k^{*}}>\frac{1}{q}-\delta / 2$, $\beta_{k^{*}}<\frac{1}{q}+\delta / 2$ and $\lambda_{k^{*}}<q \mathrm{e}^{-1 / q}+\delta / 2$ (such an $k^{*}$ exists by Lemma 3). Choose a sufficiently small constant $\zeta$ and constants $a_{1}, \ldots, a_{k^{*}}, b_{1}, \ldots, b_{k^{*}}, \ell_{1}^{(0)}, \ldots, \ell_{k^{*}}^{(\Delta)}$ such that

(i) $a_{1}=\alpha_{1}, b_{1}=\beta_{1}, \ell_{1}^{(d)}=\lambda_{1}^{(d)}$;

$$
\text { - } a_{k+1}<\mathrm{e}^{-b_{k}} / \ell_{k}^{(\Delta)}-\zeta \text {; }
$$

- $b_{k+1}>\mathrm{e}^{-a_{k}} /\left(q \mathrm{e}^{-(1+\zeta) / q}\right)+\zeta$;

- $\ell_{k+1}^{(d)}=\frac{q b_{k}-1}{b_{k}-a_{k}} \mathrm{e}^{-(1-\zeta) a_{k} d / \Delta}+\frac{1-q a_{k}}{b_{k}-a_{k}} \mathrm{e}^{-(1-\zeta) b_{k} d / \Delta}$

(iv) $a_{k^{*}}>\frac{1}{q}-\delta, b_{k^{*}}<\frac{1}{q}+\delta$. 
The existence of such constants follows easily from the continuity of the relevant functions over the region $a \neq b$.

Note that we can assume that $\rho$ is as small as we wish, as if Lemma 2 holds with $\rho=\rho_{1}$ then it clearly holds with $\rho=\rho_{2}$ for any $\rho_{2}>\rho_{1}$. In particular, we will assume that

- $1 /(q-\rho)<\mathrm{e}^{\left(1+\zeta-a_{k^{*}}\right) / q} / q$; and

- $\mathrm{e}^{-\rho /(q-1)}>\mathrm{e}^{1 / q-b_{k^{*}}}$.

We define $L_{t}(v)$ to be the set of colours available for $v$ at time $t$; we sometimes omit the subscript $t$ when it is not necessary. For any vertex $u$ and time $t$, we define $\bar{t}(u, t)$ to be the last time before $t$ that $u$ is selected. To be precise, we define $\bar{t}(u, t)$ to be zero in the event that $u$ is not selected before time $t$. This notation allows us to define a more careful refinement of the vague quantity $T(v, c)$ from subsection 1.2:

$$
T_{t}(v, c)=\sum_{w \in N(v), c \in L_{\bar{t}(w, t)}(w)} \frac{1}{\left|L_{\bar{t}(w, t)}(w)\right|} .
$$

Note that

$$
\sum_{c=1}^{C} T_{t}(v, c)=\sum_{w \in N(v)} \sum_{c \in L_{\bar{t}(w, t)}(w)} \frac{1}{\left|L_{\bar{t}(w, t)}(w)\right|}=d(v) .
$$

We will prove inductively that

Lemma 5 For each $1 \leq k \leq k^{*}$, with probability at least $1-n^{-6}$, for every $v$ with $d(v)>\rho \Delta$, colour $c$ and $30 k n \log n \leq t \leq n^{2}$, we have:

(a) $q \mathrm{e}^{-(1+\zeta) d(v) / C} \Delta \leq\left|L_{t}(v)\right| \leq \ell_{k}^{(d(v))} \Delta$;

(b) $a_{k} d(v) / \Delta \leq T_{t}(v, c) \leq b_{k} d(v) / \Delta$.

The lower bound in Part (a) is essentially Lemma 4.1 and (7) of Dyer and Frieze[2], and our proof is similar to theirs.

Proof The proof is by induction on $k$. The base case $k=1$ holds trivially. So assume that it holds for some $k$ and consider $k+1$. 
Let the neighbours of $v$ be $w_{1}, \ldots, w_{d(v)}$ and for each $i$ let $t_{i}=\bar{t}\left(w_{i}, t\right)$ be the last time before $t$ that $w_{i}$ is selected. For each $i$, let $N_{w_{i}}-v=$ $\left\{w_{i, 1}, \ldots, w_{i, d\left(w_{i}\right)-1}\right\}$ and let $t_{i, j}=\bar{t}\left(w_{i, j}, t_{i}\right)$ be the last time before $t_{i}$ that $w_{i, j}$ is selected. Similarly, for each $i, j$ let $N_{w_{i, j}}-w_{i}=\left\{w_{i, j, 1}, \ldots, w_{i, j, d\left(w_{i, j}\right)-1}\right\}$ and let $t_{i, j, r}=\bar{t}\left(w_{i, j, r}, t_{i, j}\right)$ be the last time before $t_{i, j}$ that $w_{i, j, r}$ is selected. (If $v$ lies in some cycles of length at most 6 , then some vertices will receive more than one label, but this does not create a problem.)

The idea of focussing on the colours assigned at these times was used by Dyer and Frieze [2].

With high probability, each $t_{i} \geq t-10 n \log n$. Indeed, the probability that this is not true is at most $\Delta\left(1-\frac{1}{n}\right)^{10 n \log n}<n^{-9}$. The same calculations give the same bound on the probability that some $t_{i, j}$ is less than $t-20 n \log n$ or that some $t_{i, j, r}$ is less than $t-30 n \log n$.

We start by proving part (a). Expose the values of $t_{1}, \ldots, t_{d(v)}, t_{1,1}, \ldots, t_{d(v), d\left(w_{d(v)}\right)-1}$.

It would be nice if we could say that the colours assigned to $w_{1}, \ldots, w_{d(v)}$ were independent, as that would greatly simplify our calculations. However, this is clearly not the case, since the colour assigned to $w_{i}$ has an effect on the next colour assigned to a neighbour of $w_{i}$, and this effect can propagate along a path which eventually leads to some $w_{j}$. This can be a very short path, if it goes through $v$; we will deal with such paths later. Otherwise, unless $w_{i}$ is one of the at most $O(1)$ vertices lying on a short cycle through $v$, the path must have length at least $D \log \Delta-2$. Our first concern will be such long paths.

Consider the following procedure: GLAUB(i). It follows the usual Glauber dynamics, but after step $t_{i}$, all neighbours of $w_{i}$ ignore $w_{i}$. More specifically, for each $u \in N\left(w_{i}\right), L(u)$ is the set of colours which do not appear on $N(u)-w_{i}$, and whenever $u$ is selected, $u$ is assigned a uniformly random colour from $L(u)$. Thus, for example, $w_{i}$ might have the same colour as some of its neighbours.

We use GLAUB to denote the usual Glauber dynamics, and we consider the two procedures to be coupled in that each has the same initial state and chooses the same vertex at each step. The color choice at each step is coupled maximally. 
A long path of disagreement from $w_{i}$ is a path $P$ of length at least $\ell=$ $(D / 3) \log \Delta$ beginning at $w_{i}$ and not going through $v$, such that the colour of each vertex in the path differs between GLAUB and GLAUB(i). (Such paths were used in a slightly different way by Dyer and Frieze [2] and earlier, in a different setting, by van den Berg and Steif[12].) We say that $w_{i}$ is influential if there is a long path of disagreement from $w_{i}$ or if by changing the colour assigned to $w_{i}$ at time $t_{i}$, and not changing any future colour/vertex choices in GLAUB(i), it is possible to create a long path of disagreement from $w_{i}$.

We define $B$ to be the set of neighbours $w_{i}$ such that either $w_{i}$ lies in a cycle through $v$ of length less than $D \log \Delta$ or $w_{i}$ is influential.

Lemma 6 With probability at least $1-n^{-10},|B|<\Delta / \log \Delta$.

Proof The set of neighbours which lie on a short cycle through $v$ is at most $2 \omega$. Therefore, we only have to count the influential vertices which do not lie on any short cycle through $v$. Consider such a vertex $w_{i}$; we will bound the probability that it is influential.

We will bound this probability by the probability that either $w_{i}$ is influential, or $t_{i}<t-20 n \log \Delta$. The probability of the latter is $(1-1 / n)^{20 n \log \Delta}<$ $\frac{1}{2} \Delta^{-11}$.

If $w_{i}$ is influential and $t_{i} \geq t-20 n \log \Delta$, then there is a path $P: w_{j}=$ $p_{0}, p_{1}, \ldots, p_{\ell}$, a colour $c$ to assign to $w_{i}$ at time $t_{i}$, and time steps $t-20 n \log \Delta<$ $s_{1}<\ldots<s_{\ell} \leq t$ such that at step $s_{r}, p_{r}$ is selected and assigned different colours in the two procedures.

Suppose that $P$ is the lexicographically first such path formed. Thus, at each step $s_{r}$, if the colours of some neighbour $u$ of $p_{r}$ disagree in the two procedures, then there is a path of disagreement from $u$ to $w_{j}$ which does not go through $p_{r}$. Since that path has length less than $\ell$, then $p_{r}$ and $u$ lie in a cycle of length less than $D \log \Delta$. Since $p_{r}$ lies in at most $\omega$ such cycles, there can be at most $2 \omega$ such neighbours. Thus, since $\left|L\left(p_{r}\right)\right|$ is always at least $C-\Delta=(q-1) \Delta$, the probability that $p_{r}$ chooses a different colour for each procedure, under the maximal coupling, is less than $2 \omega /((q-1) \Delta)$. Therefore, the probability that such a path is formed is at most:

$$
\Delta \times(\Delta-1)^{\ell} \times\left(\begin{array}{c}
20 n \log \Delta \\
\ell
\end{array}\right) \times\left(\frac{2 \omega}{(q-1) \Delta}\right)^{\ell}<\Delta \times\left(\frac{100 \mathrm{e} \omega}{D}\right)^{\ell}<\frac{1}{2} \Delta^{-11}
$$


for $D$ sufficiently large and $q$ near $q^{*}$. Thus, after adding the probability that $t_{i}<t-20 n \log \Delta$, the probability that $w_{i}$ is influential is at most $\Delta^{-11}$.

Now consider any collection $w_{i_{1}}, \ldots, w_{i_{m}}$ of $m=\Delta / \log \Delta-2 \omega$ neighbours which don't lie in short cycles through $v$. Using the fact that long paths of disagreement from any two such neighbours must be disjoint, similar calculations yield that the probability of all $m$ neighbours being influential is at most $\left(\Delta^{-11}\right)^{m}$. Therefore, the probability that at least $m$ such neighbours of $v$ are influential is at most

$$
\left(\begin{array}{c}
\Delta \\
m
\end{array}\right) \times \Delta^{-11 m}<\Delta^{-10 m}<n^{-10},
$$

for $D>1$.

Now, for each $i, j$, we expose the colour assigned to $u_{i, j}$ at time $t_{i, j}$. We also expose the set $B$. We denote this set of information, along with the values of $t_{1}, \ldots t_{d(v)}, t_{1,1}, \ldots t_{d(v), d\left(w_{d(v)}\right)-1}$, by $\mathcal{H}$. We say that $\mathcal{H}$ is good if every $t_{i}, t_{i, j} \geq t-30 n \log n$, if the sets of colour assignments satisfy the conditions of Lemma 5 for $k$ and if $|B| \leq \Delta / \log \Delta$. We will show by induction that the probability of $\mathcal{H}$ not being good is at most $O\left(n^{-9}\right)$.

Lemma 7 For any good $\mathcal{H}$, the conditional probability that, at a particular time $t \geq 30(k+1) n \log n$ during MOD-GLAUB, $|L(v)| \leq q \mathrm{e}^{-(1+\zeta / 2) d(v) / C} \Delta$ or $|L(v)| \geq \ell_{k+1}^{(d(v))} \Delta-\zeta / 2$ is at most $n^{-9}$.

Proof Rather than analyzing $|L(v)|$ directly, we will focus on $\left|L^{*}(v)\right|$, the set of colours which do not appear on any $w_{i} \in N(v)-B$. Note that $\left|L^{*}(v)\right| \geq|L(v)| \geq\left|L^{*}(v)\right|-|B|=\left|L^{*}(v)\right|-o(\Delta)$.

Rather than dealing with GLAUB directly, we consider the procedure MOD-GLAUB, whereby after time $t-30 n \log n$, neighbours of $v$ ignore the colour on $v$.

The advantage of focussing on $\left|L^{*}(v)\right|$ and MOD-GLAUB is that the colour assignments to the vertices in $N(v)-B$ are independent. This is because no combination of assignments to these vertices can produce a long path of disagreement from one such vertex to another, and, since the vertices ignore the colour on $v$ and no two lie on a short cycle through $v$, there are no short paths of disagreement between these vertices. 
Furthermore, exposing the fact that $w_{i} \notin B$, only exposes that $w_{i}$ is not influential. Since the definition of "influential" does not depend on the particular colour assigned to $w_{i}$, this does not expose anything about that choice of colour.

So each $w_{i}$ receives a uniformly random colour from amongst those colours which $\mathcal{H}$ dictates to not appear on $N\left(w_{i}\right)-v$ at time $t_{i}$, and the choices for $w_{1}, \ldots, w_{\Delta}$ are independent. Thus, for any colour $c$ the conditional probability that $c$ belongs to $L^{*}(v)$ at time $t$ is

$$
\prod_{w \in N(v)-B, c \in L(w)} 1-\frac{1}{L(w)}=\exp (-T(v, c))+o(1),
$$

since every $L(w)$ has size at least $C-\Delta=(q-1) \Delta$, and since $|B|=o(\Delta)$. Therefore, the expected size of $L^{*}(v)$ at time $t$ is equal to $\sum_{c} \exp (-T(v, c))+$ $o(\Delta)$.

Recall that $\sum_{c} T(v, c)=d(v)$. Furthermore, since $\mathcal{H}$ is good, each $T(v, c)$ lies between $a_{k} d(v) / \Delta$ and $b_{k} d(v) / \Delta$. Subject to these constraints, $\sum_{c} \exp (-T(v, c))$ is easily seen to be minimized when every $T(v, c)=d(v) / C$, and maximized when every $T(v, c)$ is either $a_{k} d(v) / \Delta$ or $b_{k} d(v) / \Delta$. In the latter case, the fact that there are $C=q \Delta$ different $T(v, c)$ terms and they sum to $d(v)$ implies that $\Delta\left(q b_{k}-1\right) /\left(b_{k}-a_{k}\right)$ of them are equal to $a_{k} d(v) / \Delta$ and the remaining $\Delta\left(1-q a_{k}\right) /\left(b_{k}-a_{k}\right)$ of them are equal to $b_{k} d(v) / \Delta$. This yields

$$
q \mathrm{e}^{-d(v) / C} \Delta \leq \operatorname{Exp}\left(\left|L^{*}(v)\right|\right) \leq \Delta \times\left(\frac{q b_{k}-1}{b_{k}-a_{k}} \mathrm{e}^{-a_{k} d(v) / \Delta}+\frac{1-q a_{k}}{b_{k}-a_{k}} \mathrm{e}^{-b_{k} d(v) / \Delta}\right) .
$$

Note that these calculations can be used to show that for all $d, k$,

$$
\ell_{k}^{(d)}>q \mathrm{e}^{-d / q}
$$

Since the colour choice for $w_{i}$ can affect $\left|L^{*}(v)\right|$ by at most 1 , Azuma's Inequality implies that $\left|L^{*}(v)\right|$ is highly concentrated and, in particular, that the probability of it differing from its expected value by $\Theta(\Delta)$ is $\mathrm{e}^{-\Theta(\Delta)}$. Since $d(v)>\rho \Delta$, this proves our bound on $\left|L^{*}(v)\right|$ in MOD-GLAUB.

Now we extend this bound to GLAUB. If the colour of $w_{i}$ differs in the two procedures, then at some step after $t-30 n \log n, w_{i}$ is assigned a colour that appears on $v$ and on no other neighbour of $w_{i}$. Since $\left|L\left(w_{i}\right)\right|$ is always greater 
than $C-\Delta=(q-1) \Delta$, this occurs with probability at most $((q-1) n \Delta)^{-1}$ at any one time step, so the probability that it occurs at least once is at most $30 n \log n /((q-1) n \Delta)<100 / D$.

So the expected number of vertices in $N(v)$ which are affected in this way is at most $100 \Delta / D=O(\log n)$. A simple application of the Chernoff Bounds shows that this number is highly concentrated, and so the probability that it is higher than $\Theta(\Delta)$ is at most $\mathrm{e}^{-\Theta(\Delta)}<n^{-10}$, for $\Delta \geq D \log n$ where $D$ is sufficiently large in terms of $\nu$. This proves the lemma.

Adding the probability of $n^{-10}+O\left(n^{-9}\right)$ that $\mathcal{H}$ is not good, and multiplying by the $n \times n^{2}$ choices for $v, t$ establishes part (a) of Lemma 5 .

Part (b) follows in the same manner. We define $B^{*}$ in a similar way to $B$, with the exception that a neighbour $w_{i}$ is in $B^{*}$ if (i) $w_{i} \in B$, (ii) it is possible, by changing the colour assigned to a neighbour $u$ of $w_{i}$, to form a long path of disagreement from $u$ not passing through $w_{i}$, or (iii) some neighbour $u$ of $w_{i}$ lies in a short cycle through $w_{i}$. The same analysis shows that $\operatorname{Pr}\left(\left|B^{*}\right|>\Delta / \log \Delta\right)<n^{-11}$. (An extra factor of $\Delta$ appears in the expected number calculation, and this is not enough to raise that expected number significantly.) We then restrict our attention to

$$
T_{t}^{*}(v, c)=\sum_{w \in N(v)-B *, c \in L_{\bar{t}(w, t)}(w)} \frac{1}{\left|L_{\bar{t}(w, t)}(w)\right|} .
$$

$\mathcal{H}$ exposes $B^{*}$, all times $t_{i}, t_{i, j}, t_{i, j, r}$ and the colours assigned to each $u_{i, j, r}$ at time $t_{i, j, r}$. Then we modify our procedure as follows: All vertices ignore the colours on $v, w_{1}, \ldots, w_{\Delta}$, and after time $t_{i, j}$, the neighbours of $w_{i, j}$ ignore the colour of $w_{i, j}$. If some $w_{i, j}$ is adjacent to $w_{i^{\prime}, j^{\prime}}$, then they ignore each other's colours. We refer to this modified procedure as MOD-GLAUB2.

Consider any $w_{i} \notin B^{*}$ and let $I\left(w_{i}, c\right)$ be the indicator variable that $c \in L\left(w_{i}\right)$ at time $t_{i}$. Suppose that $d\left(w_{i}\right)>\rho \Delta$. As in the proof of part (a), $\operatorname{Pr}\left(I\left(w_{i}, c\right)=1\right)=\exp \left(-T_{t_{i}}\left(w_{i}, c\right)\right)+o(1)$. Therefore, using the bounds on $T_{t_{i}}\left(w_{i}, c\right)$ and the list sizes from the fact that $\mathcal{H}$ is good, the expected value of $I\left(w_{i}, c\right) /\left|L_{t_{i}}\left(w_{i}\right)\right|$ is at most

$$
\left(\mathrm{e}^{-a_{k} d\left(w_{i}\right) / \Delta}+o(1)\right) \times \frac{1}{q \mathrm{e}^{-(1+\zeta) d\left(w_{i}\right) / C} \Delta} \leq \frac{1}{\Delta} \times \mathrm{e}^{-a_{k} / q} /\left(q \mathrm{e}^{-(1+\zeta) / q}\right)+o(1),
$$

since the fact that $a_{k}<1 / q$ implies that the LHS is maximized at $d\left(w_{i}\right)=\Delta$. 
We must take more care in proving our lower bound. We use $L$ to denote $\left|L\left(w_{i}\right)\right|$ at time $t_{i}$, we let $R$ be the event that $L \leq \ell_{k+1}^{(d(v))} \Delta$, and we let $I_{R}$ be the indicator variable for $R$. In part (a), we proved that $\operatorname{Pr}\left(I_{R}=0\right)=O\left(n^{-9}\right)$. Therefore,

$$
\begin{aligned}
\operatorname{Exp}\left(\frac{I\left(w_{i}, c\right)}{L}\right) & \geq \operatorname{Exp}\left(\frac{I\left(w_{i}, c\right)}{L} \times I_{R}\right) \\
& \geq \frac{\operatorname{Pr}\left(I\left(w_{i}, c\right)=1\right)-\operatorname{Pr}\left(I_{r}=0\right)}{\ell_{k+1}^{(d(v))} \Delta} \\
& =\frac{\operatorname{Pr}\left(I\left(w_{i}, c\right)\right)}{\ell_{k+1}^{(d(v))} \Delta}+o(1 / \Delta)
\end{aligned}
$$

Furthermore, we have

$$
\begin{aligned}
\ell_{k+1}^{d} & =\frac{1}{b_{k}-a_{k}}\left(\left(q b_{k}-1\right) \mathrm{e}^{-(1-\zeta) a_{k} d / \Delta}+\left(1-q a_{k}\right) \mathrm{e}^{-(1-\zeta) b_{k} d / \Delta}\right) \\
& <\frac{1}{b_{k}-a_{k}}\left(\left(q b_{k}-1\right) \mathrm{e}^{-(1-\zeta) a_{k}}+\left(1-q a_{k}\right) \mathrm{e}^{-(1-\zeta) b_{k}}\right) \times \mathrm{e}^{(1-\zeta) b_{k}(1-d / D)} \\
& =\ell_{k+1}^{(\Delta)} \times \mathrm{e}^{(1-\zeta) b_{k}(1-d / D)} .
\end{aligned}
$$

Thus, the expected value of $I\left(w_{i}, c\right) /\left|L\left(w_{i}\right)\right|$ at time $t$ is at least

$$
\begin{aligned}
\left(\mathrm{e}^{-b_{k} d\left(w_{i}\right) / \Delta}+o(1)\right) \times \frac{1}{\ell_{k+1}^{(d(w))} \Delta} & \geq \frac{1}{\Delta} \times \frac{\mathrm{e}^{-b_{k}}}{\ell_{k+1}^{(\Delta)}} \times \mathrm{e}^{\left(b_{k}-(1-\zeta) b_{k}\right)\left(1-d\left(w_{i}\right) / \Delta\right)}+o(1) \\
& \geq \frac{1}{\Delta} \times \frac{\mathrm{e}^{-b_{k}}}{\ell_{k+1}^{(\Delta)}}+o(1) \\
& \geq \frac{1}{\Delta} \times \frac{\mathrm{e}^{-b_{k}}}{\ell_{k}^{(\Delta)}}+o(1) .
\end{aligned}
$$

If $d\left(w_{i}\right) \leq \rho \Delta$, then $\left|L\left(w_{i}\right)\right|$ is never less than $C-\rho \Delta=(q-\rho) \Delta$. Therefore, by our assumptions on how small $\rho$ is, the expected value of $I\left(w_{i}, c\right) /\left|L\left(w_{i}\right)\right|$ is at most

$$
\frac{1}{(q-\rho) \Delta}<\frac{1}{\Delta} \times \mathrm{e}^{-a_{k} / q} /\left(q \mathrm{e}^{-(1+\zeta) / q}\right)
$$


Furthermore, since no list is ever smaller than $(q-1) \Delta, \operatorname{Pr}\left(I\left(w_{i}, c\right)\right) \geq$ $\left(1-\frac{1}{(q-1) \Delta}\right)^{\rho \Delta}$ which, by (1) and our assumptions on the size of $\rho$, is greater than $\frac{C}{\Delta} \times \mathrm{e}^{-b_{k}} / \ell_{k}^{(\Delta)}$. Therefore, the expected value of $I\left(w_{i}, c\right) /\left|L\left(w_{i}\right)\right|$ is again at least $\frac{1}{\Delta} \times \mathrm{e}^{-b_{k}} / \ell_{k}^{(\Delta)}$.

This implies that the expected value of $T_{t}^{*}(v, c)$ is at most $\left(a_{k+1}-\zeta\right) d(v) / \Delta$ and at least $\left(b_{k+1}-\zeta\right) d(v) / \Delta$. By viewing the colour assignments to $w_{i, 1}, \ldots, w_{i, \Delta-1}$ as one single random choice, we have $\Delta$ choices, each of which can affect $T_{t}^{*}(v, c)$ by at most $1 /((q-1) \Delta)$, since no $L(u)$ can have size less than $(q-1) \Delta$. Therefore by Azuma's Inequality, the probability that, under the procedure MOD-GLAUB2, $T_{v, c}$ differs from its expected value by more than $(\zeta / 2) d(v) / \Delta$ is at most $\mathrm{e}^{-\Theta(\Delta)}<n^{-10}$, for $D$ sufficiently large in terms of $\zeta$. (Again, we use the fact that $d(v) \geq \rho \Delta$.)

Virtually the same argument as that used for Part (a) proves that with sufficiently high probability, $T_{t}^{*}(v, c)$ in GLAUB is within $(\zeta / 4) d(v) / C$ of its value in MOD-GLAUB2. Furthermore, if $\mathcal{H}$ is good then $\left|T_{t}^{*}(v, c)-T_{t}(v, c)\right|<$ $(\Delta / \log \Delta) \times(1 /(q-1) \Delta)=o(1)$. This proves part (b).

Finally, we need to note that, since with probability at least $1-n^{-9}$ we have each $t_{i}, t_{i, j} \geq t-30 n \log n \geq 30 k n \log n$, then we have by induction that the probability $\mathcal{H}$ is not good is at most $2 n^{-9}$.

We now show that Lemma 2 follows from Lemma 5, upon taking $\delta$ sufficiently small when specifying $k^{*}$ and thus obtaining $a_{k}, b_{k}, \ell_{k^{*}}^{d}$ sufficiently close to $1 / q, 1 / q, q \mathrm{e}^{-d / C}$.

Proof of Lemma 2 We prove Lemma 2 in the same way as the inductive step for Lemma 5(b). We consider the procedure MOD-GLAUB2. We expose $\mathcal{H}$ and, by taking $\tau \geq 30 k^{*}$, we can assume it is (with probability at least $\left.1-O\left(n^{-6}\right)\right)$ such that for each $w_{i}$ with $d\left(w_{i}\right)>\rho \Delta$,

$$
\left(\frac{1}{q}-\delta\right) d\left(w_{i}\right) / \Delta<T\left(w_{i}, c_{1}\right), T\left(w_{i}, c_{2}\right)<\left(\frac{1}{q}+\delta\right) d\left(w_{i}\right) / \Delta,
$$

and

$$
\left|L\left(w_{i}\right)\right| \geq q \mathrm{e}^{-(1+\zeta) d(v) / C} \Delta,
$$

and that $\left|B^{*}\right|<\Delta / \log \Delta$. This last assumption, along with the fact that $|L(u)| \geq C-q$ for every vertex $u$, implies that $\sum_{w_{i} \in B^{*}}\left|L\left(w_{i}\right)\right|^{-1}=o(1)$. Thus we can restrict our attention to $N(v)-B^{*}$. 
Now consider any $w_{i} \in N(v)-B^{*}$ with $d\left(w_{i}\right)>\rho \Delta$. Let $E_{1}$ (resp. $\left.E_{2}\right)$ be the event that $c_{1}$ (resp. $c_{2}$ ) appears on $N\left(w_{i}\right)-v$. Thus $E_{1} \cap E_{2}$ is the event that $w_{i} \notin \theta$. We will estimate $\operatorname{Pr}\left(E_{1} \cap E_{2}\right)=1-\operatorname{Pr}\left(\overline{E_{1}}\right)-\operatorname{Pr}\left(\overline{E_{2}}\right)+$ $\operatorname{Pr}\left(\overline{E_{1}} \cap \overline{E_{2}}\right)$. By our assumption on $\mathcal{H}$, each $\operatorname{Pr}\left(\overline{E_{r}}\right)$ is at most

$$
\exp \left(-T\left(w_{i}, c_{r}\right)\right) \leq \exp \left(-\left(\frac{1}{q}-\delta\right) d\left(w_{i}\right) / \Delta\right) .
$$

Also, $\operatorname{Pr}\left(\overline{E_{1}} \cap \overline{E_{2}}\right)$ is at least

$$
\begin{aligned}
& \prod_{u \in N\left(w_{i}\right) ; c_{1} \in L(u) ; c_{2} \notin L(u)}\left(1-\frac{1}{|L(u)|}\right) \times \prod_{u \in N\left(w_{i}\right) ; c_{2} \in L(u) ; c_{2} \notin L(u)}\left(1-\frac{1}{|L(u)|}\right) \\
& \times \prod_{u \in N\left(w_{i}\right) ; c_{1}, c_{2} \in L(u)}\left(1-\frac{2}{|L(u)|}\right) \\
& =\exp \left(-T\left(w_{i}, c_{1}\right)-T\left(w_{i}, c_{2}\right)\right)+o(1) \\
& \geq \exp \left(-2\left(\frac{1}{q}+\delta\right) d\left(w_{i}\right) / \Delta\right)+o(1) .
\end{aligned}
$$

For $\delta$ sufficiently small in terms of $\epsilon$, this yields $\operatorname{Pr}\left(E_{1} \cap E_{2}\right)>\left(1-\mathrm{e}^{-d\left(w_{i}\right) / q \Delta}\right)^{2}-$ $\epsilon / 2$. Setting $y=\mathrm{e}^{-d\left(w_{i}\right) / q \Delta}$, note that $\left(1-(1-y)^{2}\right) /(y q \Delta)=(2-y) /(q \Delta)$ increases as $y$ decreases and so is maximized at $d\left(w_{i}\right)=\Delta$. Therefore, for $\zeta$ sufficiently small in terms of $\epsilon$, we have:

$$
\begin{aligned}
\operatorname{Exp}\left(R_{c_{1}, c_{2}}(v)\right) & \leq o(1)+\sum_{w \in N(v)} \frac{\left(1-\left(1-\mathrm{e}^{-d\left(w_{i}\right) / q \Delta}\right)^{2}\right)+\epsilon / 2}{q \mathrm{e}^{-(1+\zeta) d\left(w_{i}\right) / C} \Delta} \\
& \leq \epsilon / 2+\sum_{w \in N(v)} \frac{\left(1-\left(1-\mathrm{e}^{-d\left(w_{i}\right) / q \Delta}\right)^{2}\right)}{q \mathrm{e}^{-d\left(w_{i}\right) / C} \Delta} \\
& \leq \epsilon / 2+\frac{\left(1-\left(1-\mathrm{e}^{-1 / q}\right)^{2}\right)}{q \mathrm{e}^{-1 / q}} \times \frac{d(v)}{\Delta}
\end{aligned}
$$

It follows as in the proof of Lemma 5(b) that this sum is highly concentrated and so the probability that it differs from its expected value by more than $\epsilon / 4$ is at most $n^{-10}$ for $D$ sufficiently large.

It follows again as in the proof of Lemma $5(\mathrm{~b})$ that the probability of it differing by more than $\epsilon / 4$ from GLAUB to MOD-GLAUB is at most $n^{-10}$ for $D$ sufficiently large. This proves Lemma 2. 
We close this section with the proof of Lemma 3, thus completing the proof of Lemma 2.

Proof of Lemma 3 Recall that we can assume that $q>q^{*}$ is sufficiently small, and so we will take $q<1.49$.

It is straightforward to show that $\alpha_{k}$ is strictly increasing and $\beta_{k}$ is strictly decreasing, and so by Lemma $4, \alpha=\lim _{k \rightarrow \infty} \alpha_{k}, \beta=\lim _{k \rightarrow \infty} \beta_{k}, \lambda=$ $\lim _{k \rightarrow \infty} \lambda_{k}^{(\Delta)}$ exist. They must satisfy

$$
\begin{aligned}
\alpha & =\mathrm{e}^{-\beta} / \lambda \\
\beta & =\mathrm{e}^{-\alpha} /\left(q \mathrm{e}^{-1 / q}\right) \\
\lambda & =\frac{q \beta-1}{\beta-\alpha} \mathrm{e}^{-\alpha}+\frac{1-q \alpha}{\beta-\alpha} \mathrm{e}^{-b}
\end{aligned}
$$

We will prove that this system has no roots for $0 \leq \alpha<1 / q$. This, along with Lemma 4, implies Lemma 3.

Rearranging the first equation of our system, we get $f(\alpha)=\alpha \lambda-\mathrm{e}^{-\beta}=0$. We will bound the derivative of $f$ with respect to $\alpha$. We start by bounding

$$
g(\alpha)=1-\beta q+\beta-\alpha \beta q .
$$

Clearly $g(\alpha) \rightarrow 0$ as $\alpha \rightarrow 1 / q$. Also, noting that the derivative of $\beta$ with respect to $\alpha$ is $-\beta$, we have $g^{\prime}(\alpha)=q \beta-\beta-q \beta+q \alpha \beta=q \alpha \beta-\beta<0$ for $\alpha<1 / q$. Therefore, $g(\alpha)>0$ for $\alpha<1 / q$.

Now, using the fact that by (1) $q \mathrm{e}^{-1 / q} \leq \lambda \leq q \mathrm{e}^{-\alpha}$, we have:

$$
\begin{aligned}
f^{\prime}(\alpha)= & \lambda-\beta \mathrm{e}^{-\beta}+\frac{\alpha}{\beta-\alpha}\left((1-2 \beta q) \mathrm{e}^{-\alpha}+(\beta-q-\alpha \beta q) \mathrm{e}^{-\beta}+(\beta+1) \lambda\right) \\
\geq & \lambda-\beta \mathrm{e}^{-\beta}+\frac{\alpha \mathrm{e}^{-\beta}}{\beta-\alpha}(1-2 \beta q+\beta-q-\alpha \beta q+q \beta+q) \\
& \quad+\frac{\mathrm{e}^{-\alpha}-\mathrm{e}^{-\beta}}{\beta-\alpha}(\alpha-2 \alpha \beta q) \\
\geq & \lambda-\beta \mathrm{e}^{-\beta}+\frac{\mathrm{e}^{-\alpha}-\mathrm{e}^{-\beta}}{\beta-\alpha}(\alpha-2 \beta) \\
\geq & q \mathrm{e}^{-1 / q}-\beta \mathrm{e}^{-\beta}-\left(\mathrm{e}^{-\alpha}-\mathrm{e}^{-\beta}\right)-\beta \frac{\mathrm{e}^{-\alpha}-\mathrm{e}^{-\beta}}{\beta-\alpha}
\end{aligned}
$$




$$
\begin{aligned}
& \geq q \mathrm{e}^{-\beta}-\beta \mathrm{e}^{-\beta}-\left(\mathrm{e}^{-\alpha}-\mathrm{e}^{-\beta}\right)-\beta \mathrm{e}^{-\beta} \frac{\mathrm{e}^{-(\alpha-\beta)}-1}{\beta-\alpha} \\
& \geq q \mathrm{e}^{-\beta}-\beta \mathrm{e}^{-\beta}-\left(\mathrm{e}^{-\alpha}-\mathrm{e}^{-\beta}\right)-\beta \mathrm{e}^{-\beta}\left(1+\frac{1}{2}(\beta-\alpha)\right) \\
& =(q-2 \beta) \mathrm{e}^{-\beta}-\left(\mathrm{e}^{-\alpha}-\mathrm{e}^{-\beta}\right)-(\beta-\alpha) \beta \mathrm{e}^{-\beta} .
\end{aligned}
$$

As $\alpha \rightarrow 1 / q$, we get $\alpha \rightarrow \beta$ and so the latter two terms tend to 0 . So this allows us to bound $f^{\prime}(\alpha)$ away from 0 when $\alpha$ is close to the discontinuity at $\alpha=1 / q$. In particular, for $1.489<q<1.49$ we have $f^{\prime}(\alpha)>.01$ when $.64 \leq a<1 / q$ and so $f(\alpha)$ has no roots in that range. Having dealt with this discontinuity, it is straightforward to check that for the same range of $q$, $f(\alpha)$ has no roots in $0 \leq \alpha \leq .58$, thus proving the lemma.

\section{Path coupling and the proof of Theorem 1}

Here, we prove Theorem 1. We consider a fixed small $\epsilon, \rho$ to be named later. We begin with a burn-in period of $\tau n \log n$ steps where $\tau>30$ is as in Lemma 2. All of our analysis will assume that $\tau n \log n<t<n^{2}$. For now, we assume that for every vertex $v$, and pair of colours $c_{1}, c_{2}$, we have (i) $|L(v)| \geq C \mathrm{e}^{-d(v) / C}-\epsilon \Delta$ and (ii) $v$ has fewer than $\gamma d(v)-\epsilon \Delta$ neighbours $w$ with $d(w) \geq \rho \Delta$ and $c_{1}, c_{2} \notin L(w)$. Later we will account for the $O\left(n^{-5}\right)$ probability that this is not the case.

We use the path coupling technique of Bubley and Dyer[1]. To do so, we couple two chains $X_{0}, X_{1}, \ldots$ and $W_{0}, W_{1}, \ldots$ with arbitrary initial colourings $X_{0}, W_{0}$, and show that, with high probability, they coincide within $O(n \log n)$ steps. To prove this fact using path coupling, at any time $t$, we consider a "path" of possibly improper colourings $X_{t}=Z_{0}, Z_{1}, \ldots, Z_{h}=W_{t}$. We define this path as follows. Consider an arbitrary ordering of the vertices $v_{1}, \ldots, v_{n}$. To form $Z_{1}$ from $Z_{0}$, we change the colour of the first vertex on which $X_{t}$ and $W_{t}$ differ from its colour in $X_{t}$ to its colour in $W_{t}$. To form $Z_{2}$ from $Z_{1}$, we change the colour of the second vertex on which $X_{t}, W_{t}$ differ, and so on. Thus, $h$ is the Hamming distance between $X_{t}, W_{t}$, i.e. the number of vertices on which they differ. (If $X_{t}=W_{t}$ then $h=0$ and $X_{t}=Z_{0}=W_{t}$.)

We couple the chains as follows: We carry out a step of $Z_{0}=X_{t}$ thus

obtaining $Z_{0}^{\prime}=X_{t+1}$. Then we maximally couple a random choice for $Z_{1}$ to 
the choice for $Z_{0}$, thus obtaining $Z_{1}^{\prime}$. (Recall that, even though $Z_{r}$ may not be a proper colouring, we can apply a step of our process to it.) Repeatedly, we maximally couple $Z_{i}$ to $Z_{i-1}$ obtaining $Z_{i}^{\prime}$, finally yielding $W_{t+1}=Z_{h}^{\prime}$.

Recall that in our key Lemma 2, vertices of degree less than $\rho \Delta$ are not included in the sum $R_{c_{1}, c_{2}}(v)$. Because of this, we need to modify the notion of Hamming distance as follows.

Suppose that we are given a particular $\rho>0$. For any two (not necessarily proper) colourings $X, W$, we define their weighted Hamming distance $H^{\prime}(X, W)$ to be the number of vertices $v$ with $d(v)>\rho \Delta$ and $X(v) \neq W(v)$ plus $3 \rho$ times the number of vertices $v$ with $d(v) \leq \rho \Delta$ and $X(v) \neq W(v)$. Note that $H^{\prime}\left(X_{t}, W_{t}\right)=\sum_{i=0}^{h-1} H^{\prime}\left(Z_{i}, Z_{i+1}\right)$. Note further that $H^{\prime}\left(X_{t+1}, W_{t+1}\right) \leq$ $\sum_{i=0}^{h-1} H^{\prime}\left(Z_{i}^{\prime}, Z_{i+1}^{\prime}\right)$, since if $X_{t+1}(v) \neq W_{t+1}(v)$ then $Z_{i}^{\prime}(v) \neq Z_{i+1}^{\prime}(v)$ for at least one $i$.

We will prove that, after an $O(n \log n)$ burn-in period, the expected value of the change of the weighted Hamming distance between any pair $Z_{r}, Z_{r+1}$ is at most $-\psi / n$ for some constant $\psi>0$. Thus, the expected value of the change of the weighted Hamming distance between $X, W$ is at most $-h \times \psi / n<-\psi / n$.

To prove this, we need to know that, with high probability, the bound in Lemma 2 applies to each $Z_{r}$. So for each $0 \leq s \leq n$ and step $t$, we define $M_{t}^{s}$ to be the (possibly improper) colouring in which vertices $v_{1}, \ldots, v_{s}$ have their colour from $W_{t}$ and $v_{s+1}, \ldots, v_{n}$ have their colours from $X_{t}$. Note that, at time $t$, each $Z_{r}$ is equal to $M_{t}^{s}$ for at least one value of $s$. At time $t$, for each vertex $v$ and for each $0 \leq s \leq n$, we define $L^{s}(v)$ to be the set of colours which do not appear in $M_{t}^{s}$ on the neighbourhood of $v . L^{X}(v)=L^{0}(v)$ is the set of colours which do not appear in $X$ on $N(v)$, and $L^{W}(v)=L^{n}(v)$ is the set of colours which do not appear in $W$ on $N(v)$.

Lemma 8 For every $\epsilon, \rho>0$, there exist constants $D, \tau$ such that with probability at least $1-n^{-5}$, for every vertex $v$, colours $c_{1}, c_{2}, 0 \leq s \leq n$ and time $\tau n \log n \leq t \leq n^{2}$, we have the following: Define $\theta=\theta_{c_{1}, c_{2}}^{s}(v)$ to be the set of neighbours $w$ of $v$ with $d(w) \geq \rho \Delta$ and with at least one of $c_{1}, c_{2}$ not appearing in $M_{t}^{s}$ on $N(w)-v$, and define:

$$
R_{c_{1}, c_{2}}^{s}(v)=\sum_{w \in \theta} \frac{1}{|L(w)|}
$$


Then

$$
R_{c_{1}, c_{2}}^{s}(v) \leq \frac{1-\left(1-\mathrm{e}^{-1 / q}\right)^{2}}{q \mathrm{e}^{-1 / q}} \times \frac{d(v)}{\Delta}+\epsilon
$$

To prove Lemma 8, we define

$$
T_{t}^{s}(v, c)=\sum_{v_{j} \in N(v) ; j \leq s ; c \in L} \sum_{\bar{t}\left(v_{j}, t\right)}^{W}\left(v_{j}\right)=\frac{1}{\left|L_{\bar{t}\left(v_{j}, t\right)}^{W}\left(v_{j}\right)\right|}+\sum_{v_{j} \in N(v) ; j>s ; c \in L_{\bar{t}\left(v_{j}, t\right)}^{X}\left(v_{j}\right)} \frac{1}{\left|L_{\bar{t}\left(v_{j}, t\right)}^{X}\left(v_{j}\right)\right|},
$$

and we modify Lemma 5 to:

Lemma 9 For each $1 \leq k \leq k^{*}$, with probability at least $1-n^{-5}$, for every $v$ with $d(v)>\rho \Delta$, colour $c, 0 \leq s \leq n$ and $30 k n \log n \leq t \leq n^{2}$, we have at time $t$ :

$$
\begin{aligned}
& \text { (a) } q \mathrm{e}^{-(1+\zeta) d(v) / C} \Delta \leq\left|L^{X}(v)\right|,\left|L^{W}(v)\right| \leq \ell_{k}^{(d(v))} \Delta ; \\
& \text { (b) } a_{k} d(v) / \Delta \leq T_{t}^{s}(v, c) \leq b_{k} d(v) / \Delta
\end{aligned}
$$

The proof of part (a) is essentially the same as in Lemma 5. To prove part (b), for each $w_{i}=v_{j}, \mathcal{H}$ exposes the colours that $w_{i, 1,1}, \ldots, w_{i, \Delta-1, \Delta-1}$ receive in the chain $W$ if $j \leq s$, and exposes the colours they receive in $X$ otherwise. The rest of the proof is the same. The exponent of $n$ in the probability bound changes from -6 to -5 because of the extra $n$ choices for $s$.

Then we prove Lemma 8 from Lemma 9 by defining $\mathcal{H}$ in the same way.

For now we assume that for every vertex $v, 0 \leq s \leq n$ and pair of colours $c_{1}, c_{2}$, we have

$$
R_{c_{1}, c_{2}}^{s}(v) \leq \frac{1-\left(1-\mathrm{e}^{-1 / q}\right)^{2}}{q \mathrm{e}^{-1 / q}} \frac{d(v)}{\Delta}+\epsilon .
$$

Later we will account for the $O\left(n^{-5}\right)$ probability that this is not the case.

Now, consider any $Z_{r}$ and $Z_{r+1}$. They differ on exactly one vertex, say $v$ which has colour $c_{1}$ in $Z_{r}$ and $c_{2}$ in $Z_{r+1}$. We apply one step of our process to $Z_{r}$ and to $Z_{r+1}$, coupled as described in the introduction. 
Case 1: $d(v)>\rho \Delta$.

The weighted Hamming distance between $Z_{r}$ and $Z_{r+1}$ decreases by 1 iff we select $v$. This has probability $1 / n$ of occurring. The weighted Hamming distance increases iff we choose a neighbour $u$ of $v$, and assign it colour $c_{1}$ in $Z_{r}$ and/or assign it colour $c_{2}$ in $Z_{r+1}$. If $d(u)>\rho \Delta$ then it increases by 1 , otherwise it increases by $3 \rho$. Thus, to increase by 1 , we must choose a neighbour $u \in \theta$ and so the probability that it increases by 1 is $R_{c_{1}, c_{2}}(v) / n$. The probability that it increases by $3 \rho$ is at most $d(v) /(n(C-\Delta))$. Therefore, the expected change in the Hamming distance is at most

$$
\frac{1}{n} \times\left(-1+\frac{1-\left(1-\mathrm{e}^{-1 / q}\right)^{2}}{q \mathrm{e}^{-1 / q}-\epsilon}+\epsilon+\frac{3 \rho}{q-1}\right)
$$

which is negative if we choose $\epsilon$ and $\rho$ to be sufficiently small in terms of $q$, since we chose $q$ such that $\left(1-\mathrm{e}^{-1 / q}\right)^{2}+q \mathrm{e}^{-1 / q}>1$.

Case 2: $d(v) \leq \rho \Delta$.

The weighted Hamming distance decreases by $3 \rho$ with probability $\frac{1}{n}$ and it increases with probability at most $\rho \Delta /(n(C-\Delta))$. Since it never increases by more than 1 , the expected change in the Hamming distance is at most

$$
\frac{1}{n} \times\left(-3 \rho+\frac{\rho}{q-1}\right)
$$

which is negative for $\epsilon$ sufficiently small since $q-1>1 / 3$ for $q>q^{*}$.

Thus, in either case, the expected change in the weighted Hamming distance is less than $-\psi / n$ for some $\psi=\psi(q)>0$. This implies that with sufficiently high probability, the weighted Hamming distance drops to 0 within $O(n \log n)$ steps.

Now we still have to account for the $O\left(n^{-5}\right)$ chance that for some $v, t, s, c_{1}, c_{2}$, $R_{c_{1}, c_{2}}^{s}(v)$ is too large. Let $t^{*}$ be the first time at which this occurs. Consider the random variable $H^{*}(t)$ defined as follows. Until time $t^{*}, H^{*}(t)=$ $H^{\prime}\left(X_{t}, W_{t}\right)$. After time $t^{*}$, if $H^{*}(t-1)>0$ then $H^{*}(t)=H^{*}(t-1)-1$ with probability $\frac{1}{n}$ and $H^{*}(t)=H^{*}(t-1)+1$ with probability $\frac{1-\psi}{n}$; if $H^{*}(t-1)=0$ then $H^{*}(t)=0 . H^{*}(t)$ is a simple random walk with negative drift after time $t=\tau n \log n$, and it is straightforward to verify that with high probability, $H^{*}(t)=0$ when $t=O(n \log n)$. Furthermore, with probability $1-O\left(n^{-4}\right)$, 
$H^{\prime}\left(X_{t}, W_{t}\right)=H^{*}(t)$ for each $1 \leq t \leq n^{2}$. Therefore, with high probability, $H^{\prime}\left(X_{t}, W_{t}\right)=0$ when $t=O(n \log n)$. This is enough to prove Theorem 1 . See, for example, [4] for the standard argument.

\section{Some final comments}

In this section, we note that this coupling argument cannot be used for the case $C=q \Delta$ for any $q<q^{*}$. To see this, consider any polynomial $n^{x}$ and any $\Delta$-regular graph $G$ with $\Delta \geq D \log n$ and girth at least $D \log \Delta$ for some sufficiently large $D$ in terms of $x$.

Note that Lemmas 2 to 9 hold for all $q>1.489$ (and in fact, if needed, we could show that they hold for even smaller $q$ ). The only place where we required $q>q^{*}$ was in the Proof of Theorem 1 in Section 3. Furthermore, the upper bound $t \leq n^{2}$ in their statements can be easily increased to $n^{x}$. Therefore, if the Glauber dynamics mixes rapidly, then a "typical" colouring will satisfy that for all $v, c,\left|L_{v}\right|$ is arbitrarily close to $C \mathrm{e}^{-1 / q}$ and $T(v, c)$ is arbitrarily close to $1 / q$. Thus, such a colouring must exist, call it $\Psi$.

Suppose that we choose $\Psi$ as our initial colouring. Then for the first $n^{x}$ steps, with high probability, for all $v, c,\left|L_{v}\right|$ is arbitrarily close to $C \mathrm{e}^{-1 / q}$ and $T(v, c)$ is arbitrarily close to $1 / q$. This implies that with high probability, for any $v, c_{1}, c_{2}$, the number of neighbours of $v$ which have either $c_{1}$ or $c_{2}$ in their list is arbitrarily close to $\left(1-\left(1-\mathrm{e}^{-1 / q}\right)^{2}\right) \Delta$. So if we couple the colouring arising at any time less than $n^{x}$ with another colouring which differs in exactly one vertex, then since $q<q^{*}$, the expected change in their Hamming distance will be positive.

Furthermore, since the graph has large girth, we cannot apply the technique from [5] and [9] where by analyzing the expected total change over a few steps, we were able to get some gain from edges in $N(v)$.

So in an extended abstract of this paper[10] the author raised the following question:

Question: Is there any $q<q^{*}$ and $D>0$ such that the Glauber dynamics for $q \Delta$-colourings mixes in polytime on graphs with girth at least $D$ and maximum degree $\Delta$ at least $D \log n$ ? 
He noted that a positive answer would require a substantial new idea. Very recently, Hayes and Vigoda[6] provided such an answer, proving that any $q>1$ will do even when the girth is as small as 9 . Their substantial new idea was to use a "non-Markovian coupling". We refer the reader to their paper for a further description.

Acknowledgements: The author is grateful to Martin Dyer and Alan Frieze for some helpful discussions, for providing him with an early copy of their paper [2], and for pointing out an error in an early version. He is also grateful to two anonymous referees for suggestions that greatly improved the presentation.

\section{References}

[1] R. Bubley and M. Dyer. Path coupling: a technique for proving rapid mixing in Markov chains. Proceedings of the 28th Annual Symposium on Foundations of Computer Science (1997), 223 - 231.

[2] M. Dyer and A. Frieze. Randomly colouring graphs with bounds on girth and maximum degree. Proceedings of the 32nd Annual Symposium on Foundations of Computer Science (2001).

[3] M. Dyer, L. Goldberg, C. Greenhill, M. Jerrum and M. Mitzenmacher, An extension of path coupling and its application to the Glauber dynamics for path colourings, Proceedings of the 11th Annual ACM-SIAM Symposium on Discrete Algorithms (2000), 355 - 363.

[4] M.Dyer and C. Greenhill. Random walks on combinatorial objects. Surveys in Combinatorics, 1999, eds. J.D. Lamb and D.A. Preece. Cambridge University Press, 1999, 101 - 136.

[5] M. Dyer, C. Greenhill and M. Molloy. Very rapid mixing of the Glauber dynamics for proper colourings on bounded-degree graphs. Random Structures and Algorithms (to appear).

[6] T. Hayes and E. Vigoda. A non-markovian coupling for sampling colourings. Preprint. 
[7] M. Jerrum, A very simple algorithm for estimating the number of $k$ colourings of a low-degree graph. Random Structures and Algorithms 7 (1995), $157-165$.

[8] M. Jerrum, Mathematical foundations of the Markov chain Monte Carlo method. Probabilistic Methods for Algorithmic Discrete Mathematics, eds. M. Habib, C. McDiarmid, J. Ramirez-Alfosin and B. Reed. Springer, 1998, $116-165$.

[9] M. Molloy. Very rapidly mixing Markov Chains for $2 \Delta$-colourings and for independent sets in a 4-regular graph. Random Struc. \& Alg. 18, $101-115$ (2001).

[10] M. Molloy. The Glauber dynamics on the colourings of a graph with large girth and maximum degree. Proceedings of STOC 2002.

[11] J. Salas and A. Sokal. Absence of phase transition for antiferromagnetic Potts models via the Dobrushin uniqueness theorem. J. Statistical Physics 86, 551 - 579 (1997).

[12] J. van den Berg and J. Steif. Percolation and the hard-core lattice gas model. Stochastic Processes and their Applications 49 (1994), 179 - 197.

[13] E. Vigoda. Improved bounds for sampling colourings. J. Mathematical Physics 41 (2000), 1555 - 1569. 